\title{
Microbiome analysis reveals gut microbiota alteration of early-weaned Yimeng black goats with the effect of milk replacer and age
}

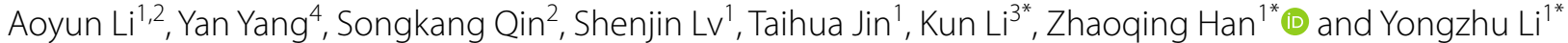

\begin{abstract}
Background: Colonization of intestinal microbiota in ruminant during the early life is important to host health, metabolism and immunity. Accumulating evidence revealed the ameliorative effect of milk replacer administration in the gut microbial development of early-weaned ruminants. Yimeng black goats (YBGs) inhabiting Shandong, China show a complex intestinal microbial ecosystem, but studies of their gut microbiota are still insufficient to report. Here, this study was performed to investigate how the gut microbiota develops in weaned YBGs with the effect of age and milk replacer.
\end{abstract}

Results: Results indicated that both age and milk replacer were important factors to change the gut microbiota of YBGs. Although the alpha diversity of gut microbiota did not change with the age of YBGs, the taxonomic compositions significantly changed. The relative abundance of some beneficial bacteria such as Lachnospiraceae, Ruminococcaceae, Ruminiclostridium, Eubacterium and Barnesiella significantly decreased and subsequently increase with age, which contributes to maintain the stability of intestinal environment and realize the diversity of intestinal functions. The relative abundance of Porphyromonas, Brevundimonas, Flavobacterium, Stenotrophomonas, Propionibacterium, Acinetobacter, Enterococcus and Clostridium belong to pathogenic bacteria in milk replacer-treated YBGs was significantly decreased. Additionally, some beneficial bacteria such as Ruminococcus, Ruminococcaceae, Christensenellaceae and Ruminiclostridium also display a trend of decreasing first followed by gradually increasing.

Conclusions: This study first revealed the gut bacterial community alterations in YBGs with the effect of age and milk replacer. This study also characterized the gut microbial distribution in YBGs with different ages and provided better insight into microbial population structure and diversity of YBGs. Moreover, milk replacer may serve as a good applicant for improving gut microbial development in early-weaned YBGs.

Keywords: Milk replacer, Gut microbiota, Yimeng black goats, Weaned, Age

*Correspondence: lik2014@sina.com; hanzhaoqing@lyu.edu.cn; liyongzhu@lyu.edu.cn

${ }^{1}$ College of Agriculture and Forestry Science, Linyi University, Linyi, China

${ }^{3}$ Institute of Traditional Chinese Veterinary Medicine, College

of Veterinary Medicine, Nanjing Agricultural University, Nanjing 210095, China

Full list of author information is available at the end of the article

\section{Introduction}

Ruminant gut microbiota composed of trillions of microorganism is the most complicated and largest microecosystem, which plays vital roles in mucosal immunity, nutrient absorption and intestinal epithelium differentiation $[1,2]$. Furthermore, the gut microbiota may also serves as a contributing or central factor of various diseases, affecting both near and far organ systems [3]. Generally, these microorganisms inhabiting in gut such as bacteria, fungi and archaea can interact in a commensal,

(c) The Author(s) 2021. This article is licensed under a Creative Commons Attribution 4.0 International License, which permits use, sharing, adaptation, distribution and reproduction in any medium or format, as long as you give appropriate credit to the original author(s) and the source, provide a link to the Creative Commons licence, and indicate if changes were made. The images or other third party material in this article are included in the article's Creative Commons licence, unless indicated otherwise in a credit line to the material. If material is not included in the article's Creative Commons licence and your intended use is not permitted by statutory regulation or exceeds the permitted use, you will need to obtain permission directly from the copyright holder. To view a copy of this licence, visit http://creativeco mmons.org/licenses/by/4.0/. The Creative Commons Public Domain Dedication waiver (http://creativecommons.org/publicdomain/ zero/1.0/) applies to the data made available in this article, unless otherwise stated in a credit line to the data. 
symbiotic or parasitic relationship resulting in stabilizing and maintaining the intestinal environment $[4,5]$. The stabilized gut bacterial community is the precondition for host performing the normal physiological functions, metabolism and immune function, but gut microbiota imbalance may result in multiple gastrointestinal diseases, such as diarrhea and irritable bowel syndrome $[6$, 7]. Ruminants displayed unique digestive properties and microbial population that enables them to evolve special adaptations in high fiber content foods, but also make them susceptible to multiple gastrointestinal diseases [8]. Given the importance of ruminant gut microbiota in many physiological functions, investigating the development and composition of its microbial community is of great significance.

Milk replacer is the artificial milk produced by replacing milk protein with non-milk protein on the basis of the nutritional standards of breast milk [9]. The nutritional components and physical form of milk replacer are similar to the breast milk, and its quality is not easily affected by the external environment [10]. Several evidence demonstrated that milk replacer administration during the ruminant juvenile period could improve the immunity and decrease the stress response caused by the sudden changes of feed morphology [11]. Moreover, milk replacer administration could also ameliorate the growth performance of kids and reduce the morbidity and mortality caused by insufficient early nutritional intake [12]. Remarkably, several recent studies indicated the beneficial effects of milk replacer administration in the early-weaning calves and kids for regulating their gut microbiota [13]. Yimeng black goats (YBGs) are an indigenous breed of the Shandong, China characterized by outstanding adaptability and stress resistance [13-15]. However, the quantity of YBGs is relatively small because of low fertility rate and delayed growth. Our previous research has demonstrated the ameliorative effect of milk replacers on the growth performance and rumen microbiota of YBGs [14]. However, studies regarding the influence of milk replacer on gut microbiota in YBGs have been insufficient to date. Taking advantage of this gap, we investigated the variability of gut microbiota in the YBGs with the effect of age and milk replacer.

\section{Materials and methods}

\section{Animals and sample acquisition}

A group of 24 one-day-old healthy YBGs with similar initial weight were obtained from a commercial feedlot (Shandong, China). The YBGs purchased for this experiment were self-propagated by the commercial feedlot and had similar genetic backgrounds. All the YBGs were randomly divided into control group (B group) and milk replacer administration group ( $\mathrm{R}$ group). The YBGs were raised in experimental animal center, Linyi University (Shandong, China) for 75 days and provided with the recommended clean environment and breeding temperature. The control and experimental groups were compulsively weaned on day 10 , but the experimental group was provided with milk replacer after weaning. Moreover, adequate starter feed and water were provided ad libitum from day 15. The nutrient composition of milk replacer and starter feed was based on our previous research [14]. Three YBGs from each group were randomly selected to sacrifice for obtaining the intestinal samples on days 15 , 25, 45 and 75. All the YBGs were euthanized by injecting pentobarbital $(25 \mathrm{mg} / \mathrm{kg})$. After euthanizing, the intestines were stripped from the mesentery by using sterilized surgical knife. The intestines (duodenal, ileum, jejunal and cecum) were knotted using cotton ropes to minimize the potential cross-contamination among the different intestines. After that, the contents from the intermediate areas of the different intestines were carefully collected. The collected intestinal samples were immediately stored in the sterilized tubes, snap-frozen using liquid nitrogen and stored at $-80{ }^{\circ} \mathrm{C}$ for further study.

\section{DNA extraction and illumina MiSeq sequencing}

Intestinal content samples were subjected to bacterial genomic DNA extraction via using QIAamp DNA Mini Kit (QIAGEN, Hilden, Germany) according to the suggested instructions of manufacturer. The integrity and size of collected DNA was verified by $0.8 \%$ agarose gel electrophoresis and the UV-Vis spectrophotometer (NanoDrop 2000, United States) was used for determining the DNA concentrations. The primers (338F: ACT CCT ACG GGA GGC AGC A and 806R: GGA CTA CHV GGG TWT CTA AT) with adaptors, which were designed according to the conserved region, were used for amplifying the V3/V4 regions. PCR amplification was performed in triplicated as described previously. The evaluation and purification of PCR amplification products were performed by using $2.0 \%$ agarose gel electrophoresis and AxyPrep DNA Gel Extraction Kit (Axygen, CA, USA), respectively. Purified PCR products were fluorescently quantified on Microplate reader (BioTek, FLx800) on the basis of the initial quantitative results of electrophoresis. Subsequently, each sample was mixed in corresponding proportion based on the fluorescence quantitative results and the sequencing quality requirements. The purified PCR amplification products were used for generating the sequencing library using Illumina TruSeq (Illumina, United States) according to manufacturer's specifications. The sequencing libraries were conducted quality inspection and fluorescence quantification prior to sequencing. The qualified libraries shown only a 
single peak and the concentration is more than $2 \mathrm{nM}$. The collected libraries were assembled, diluted and mixed in proportion based on the quantity of sequencing required. Finally, the libraries were performed high-throughput sequencing by using MiSeq sequencing machine.

\section{Bioinformatics and data analysis}

The paired-end sequences obtained from sequencing were merged into a tag and the quality of raw reads were screened. Specifically, the reads of each sample were spliced through overlap using FLASH software (v1.2.7) to obtain original tags. Moreover, the Trimmomatic (v0.33) software and UCHIME (v4.2) software were used to filter the original tags and eliminated chimera, respectively to achieve effective Tags. The obtained high-quality sequences were clustered to the same OTU on the basis of $97 \%$ similarity. Representative sequence of each OTU was performed classification identification and phylogenetic analysis. The alpha diversity was calculated based on the abundance distribution of OTUs in different samples. Beta diversity was performed using QIIME (Version 1.7.0) to compare the difference and similarity among different samples. Moreover, the rarefaction curves were constituted to assess the sequencing depth. Linear discriminant analysis effect size (LEfSe) was generated to analyse the differentially abundant taxon. R (v3.0.3) and GraphPad Prism (version 7.0c) were applied to statistical analysis. P-values $<0.05$ were considered statistically significant and the data was expressed as means $\pm S D$.

\section{Results}

\section{Sequences analyses}

Following taxonomic assignment, a total of 11,789 OTUs were recognized based on $97 \%$ nucleotide-sequence similarity with an average of 245 OTUs per sample. On average, $3898,2379,3228$ and 2284 OTUs belonged to control jejunum, control cecum, milk replacer treated jejunum, milk replacer treated cecum, respectively (Fig. 1a-d). Moreover, we also observed 41 core OTUs in all the jejunum as well as 334 core OTUs were identified in all the cecum (Fig. 1e, f). The rank abundance curve of each sample is wide and falls gently, displaying the excellent evenness and richness (Fig. 1g). Moreover, both OTU curve and species accumulation curve were relatively flat and displayed a tendency to saturate as the number of qualified sequences exceed 20,000, indicating that the sequencing quantity and depth meet the requirement for sequencing and analysis (Fig. 1h, i).

\section{Alterations in gut microbial diversities with the effect of milk replacer and age}

To assess the differences in the diversity and abundance of gut microbiota between different groups the qualified sequences achieved in the sequencing were aligned to estimate alpha index. Alpha diversity of gut microbial population could be reflected by community abundance (Chao1), diversity index (Shannon and Simpson) and sequencing depth (Good's coverage). Good's coverage estimates in all the groups were approximately 100\%, implying the excellent coverage (Fig. 2a, e). We observed that Chao1, Simpson and Shannon indices did not change significantly with age, implying that the diversity and abundance in intestinal microbiota of YBGs did not change from days 15 to 75 (Fig. 2b-d). Moreover, there was no statistically distinct difference in three diversity indices between both groups, indicating that milk replacer administration had no effect on the abundance and diversity of the intestinal microbiota of YBGs (Fig. 2f-h).

The PCoA plots based on the weighted UniFrac distances indicated that despite of shared diets and growing environment, the YBGs displayed continuous alterations in their gut microbial communities with age (Fig. 3a). However, the individuals in same group were clustered together, indicating that the intestinal microbiota composition between the samples in one group was similar. PCoA plots also revealed a separation of the jejunum and cecum, suggesting that the principal compositions of gut microbiota between jejunum and cecum were significantly different. Moreover, the samples in both groups gradually clustered with time, indicating that milk replacer administration had no effect on the main compositions of gut microbiota (Fig. 3b).

\section{Significant alterations in the gut microbial compositions with the age of YBGs}

The relative proportion of preponderant taxa at the levels of phylum and genus were assessed through microbial taxa assignment in different groups (Fig. 4). According to the phylum assignment result, phyla Firmicutes, Proteobacteria and Bacteroidetes were the most dominant bacteria in the control YBGs regardless of age, accounting for approximately $90 \%$ of the taxonomic groups identified. Additionally, bacteria belonging to the phyla Synergistetes, Verrucomicrobia, Tenericutes and Actinobacteria were represented with a lower abundance in all the samples. At the genus level, Bacteroides (15.28\%), Lactobacillus (9.95\%) and Prevotella_1 (5.61\%) were the three most dominant genera in the jejunum of 15-dayold YBGs, whereas Shewanella (5.54\%), Sporolactobacillus (3.02\%) and Lactobacillus (1.33\%) were observed as the predominant in the jejunum of 25-day-old YBGs. Moreover, the most abundant genera were unidentified_Chloroplast (16.40\%), Alloprevotella (15.48\%) and Pseudomonas (8.76\%) in the jejunum of 45-day-old YBGs, while the occurrence of Lactobacillus (29.62\%), 

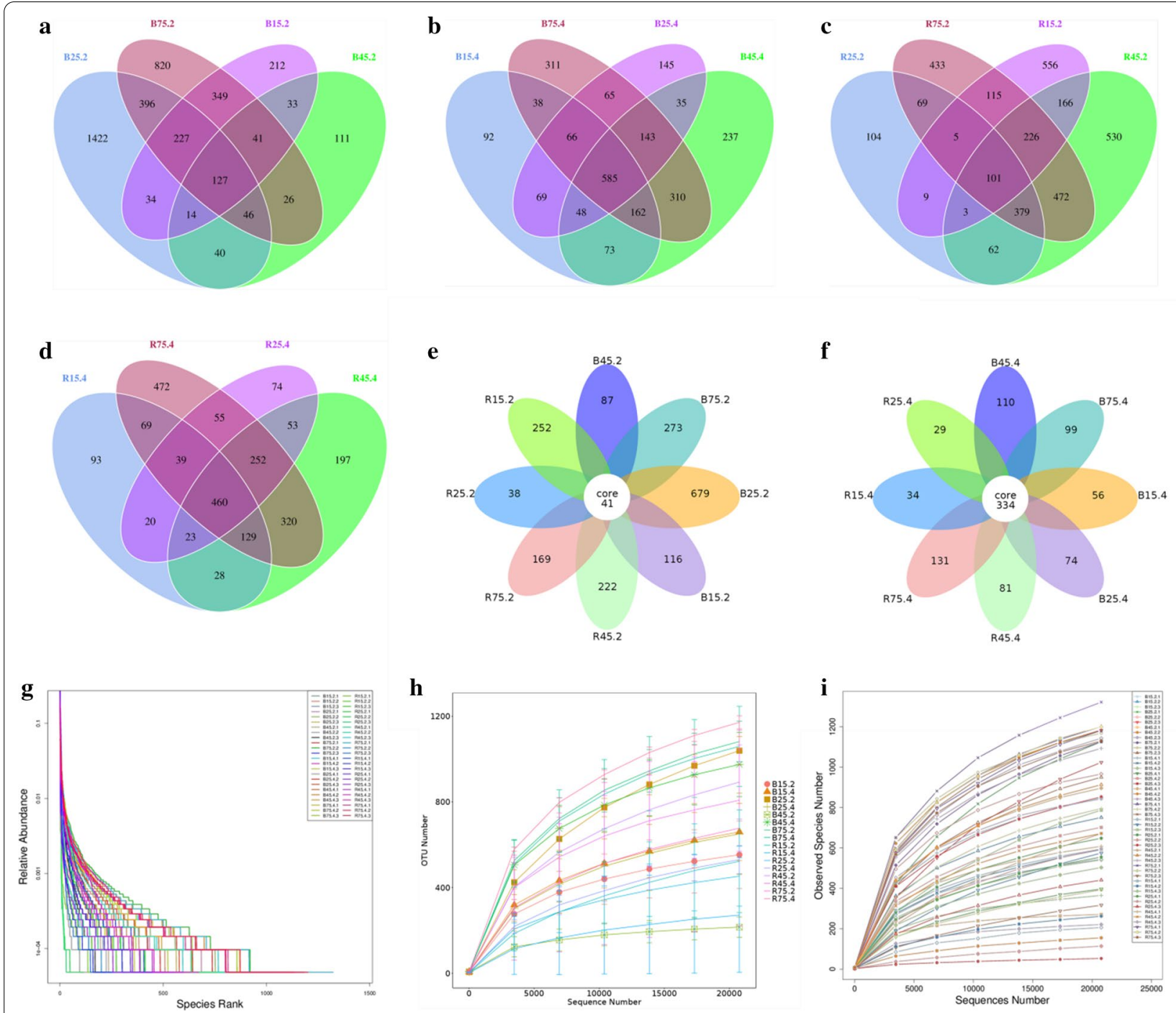

Fig. 1 Operational taxonomic units (OTUs) and sample feasibility analysis. Venn diagrams show unique and shared bacterial OTUs in jejunum (a) the jejunum in control group; (c) the jejunum in milk replacer-treated group) and cecum (b) the cecum in control group, (d) the cecum in milk replacer-treated group). Venn diagrams for core OTUs distribution in all the jejunum (e) and cecum (f). Rank-Abundance (g) and rarefaction curves (h, i) were used for the assessment of sequencing depth. Each color-curve represents a sample. B15.2, B25.2, B45.2 and B75.2 represent the jejunum in the control group on the days 15, 25, 45 and 75, respectively. B15.4, B25.4, B45.4 and B75.4 represent the cecum in the control group on the days 15, 25, 45 and 75, respectively. R15.2, R25.2, R45.2 and R75.2 indicate the jejunum in the milk replacer-treated group on the days $15,25,45$ and 75 , respectively. R15.4, R25.4, R45.4 and R75.4 indicate the cecum in the milk replacer-treated group on the days 15, 25, 45 and 75, respectively

Prevotella_1 (58.13\%) and Cetobacterium (4.48\%) was higher in the jejunum of 75-day-old YBGs. Lactobacillus (25.58\%) and Ruminococcaceae_UCG-005 (14.73\%) were the most predominant bacterium in the cecum of 15-day-old and 45-day-old YBGs, followed by Bacteroides $(12.45 \%, 8.58 \%)$ and Escherichia-Shigella $(5.64 \%$, $6.05 \%$ ), which together made up $43.67 \%$ and $29.36 \%$ of the overall bacterial composition, respectively. Moreover, Bacteroides (30.99\%), Peptoclostridium (5.14\%) and Escherichia-Shigella $(4.86 \%)$ were the most prevalent bacteria in the cecum of 25-day-old YBGs, whereas Bacteroides (27.30\%), Chlamydophila (7.33\%) and Prevotella_1 (5.55\%) were observed predominant in the cecum of 75-day-old YBGs. Moreover, The distribution of bacterial genera in each sample can also be observed in the heatmap (Fig. 5).

Using LEfSe analysis to compare the bacterial genus-level taxonomic compositions among groups, we found that the relative abundances of Pseudobutyrivibrio, Eubacterium_ventriosum_group and 

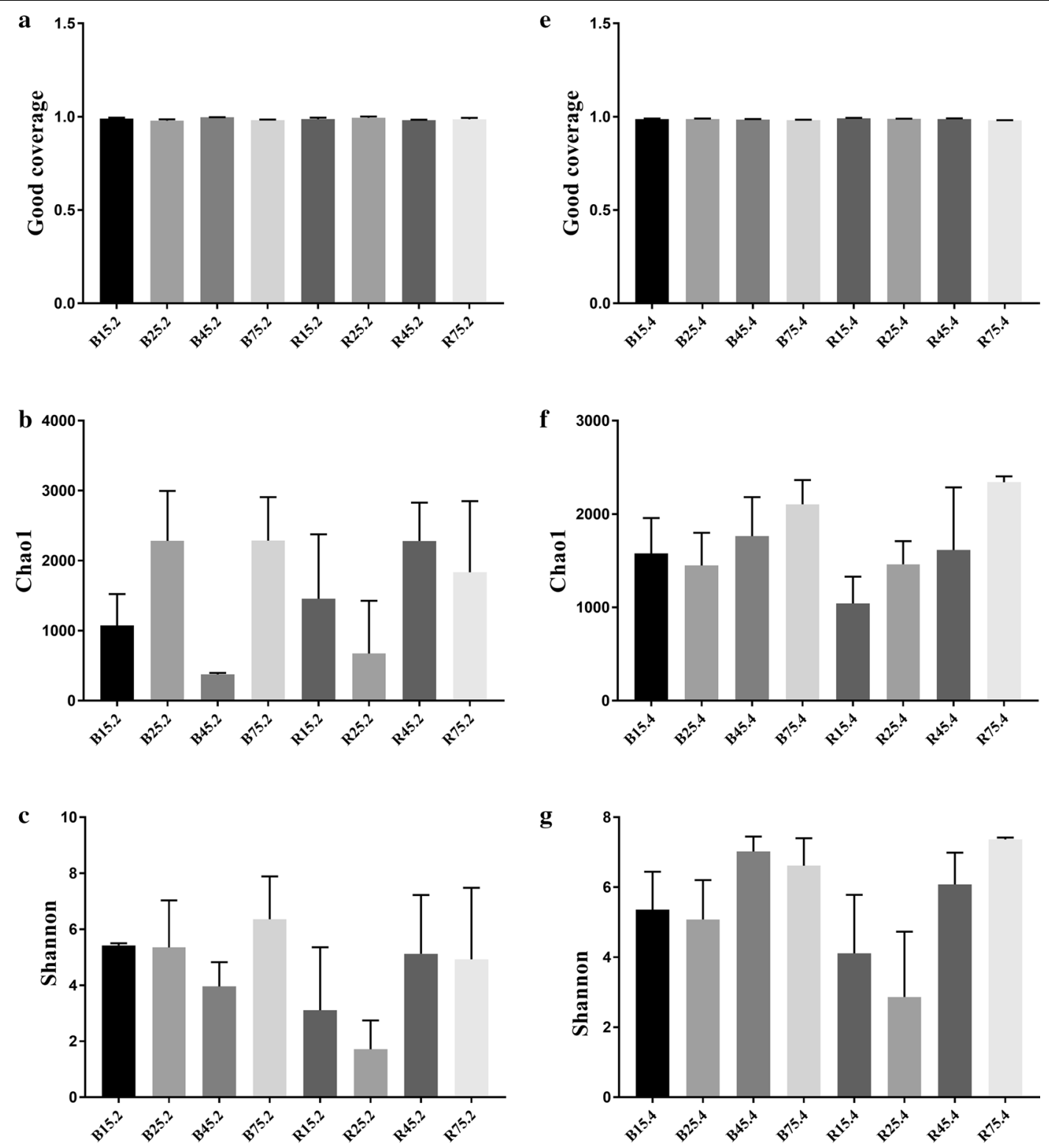

g
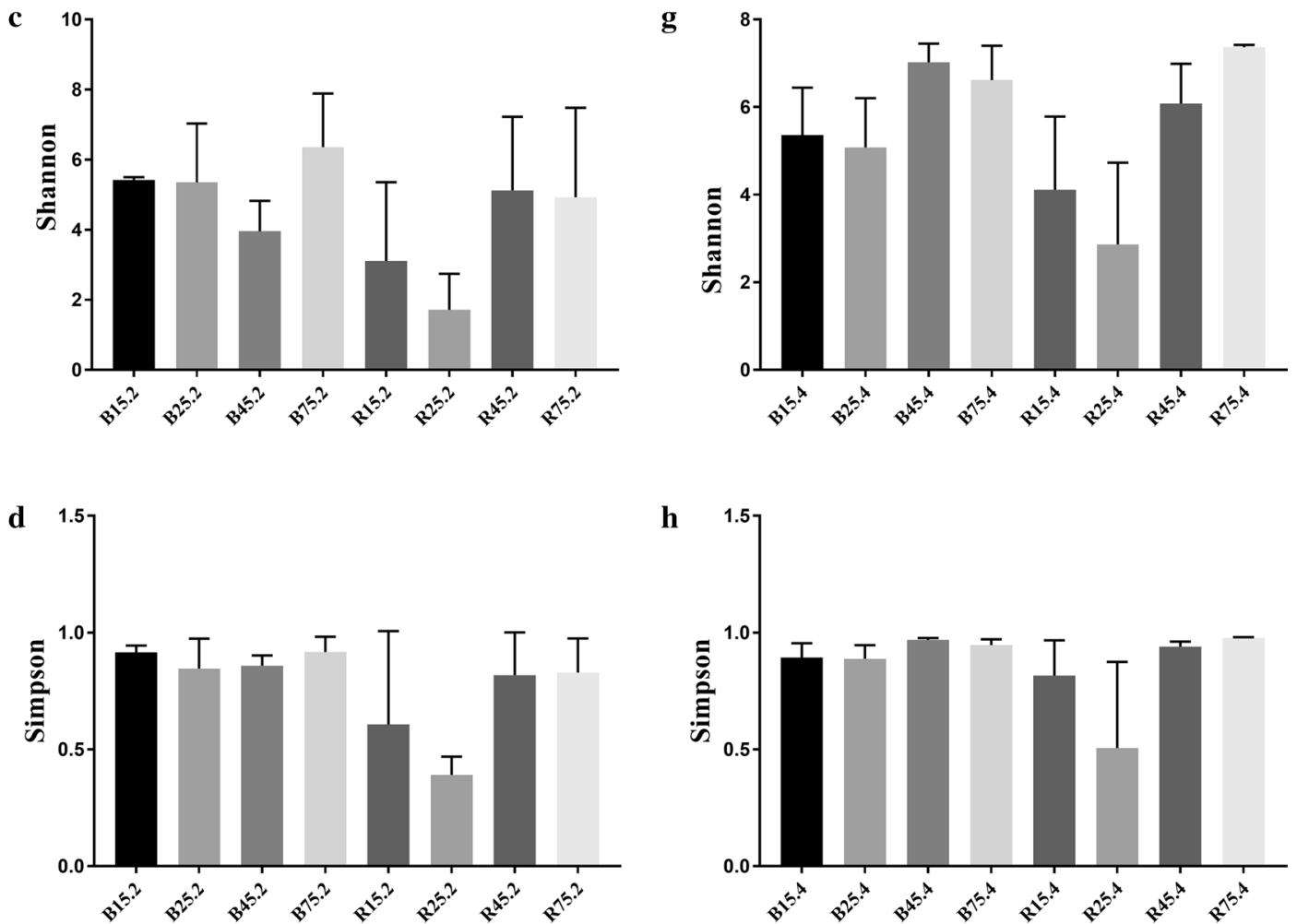

h

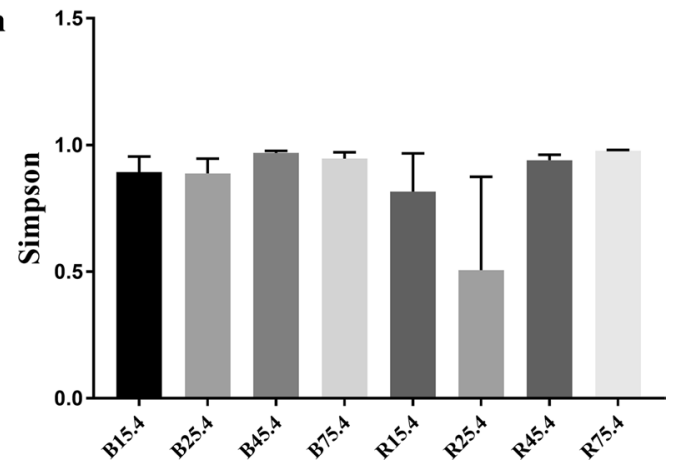

Fig. 2 Microbial diversity index analysis. Good's coverage (a,e), Chaol (b, f), Shannon (c, g) and Simpson (d, h) were applied to assess the alpha diversity of gut microbial community 

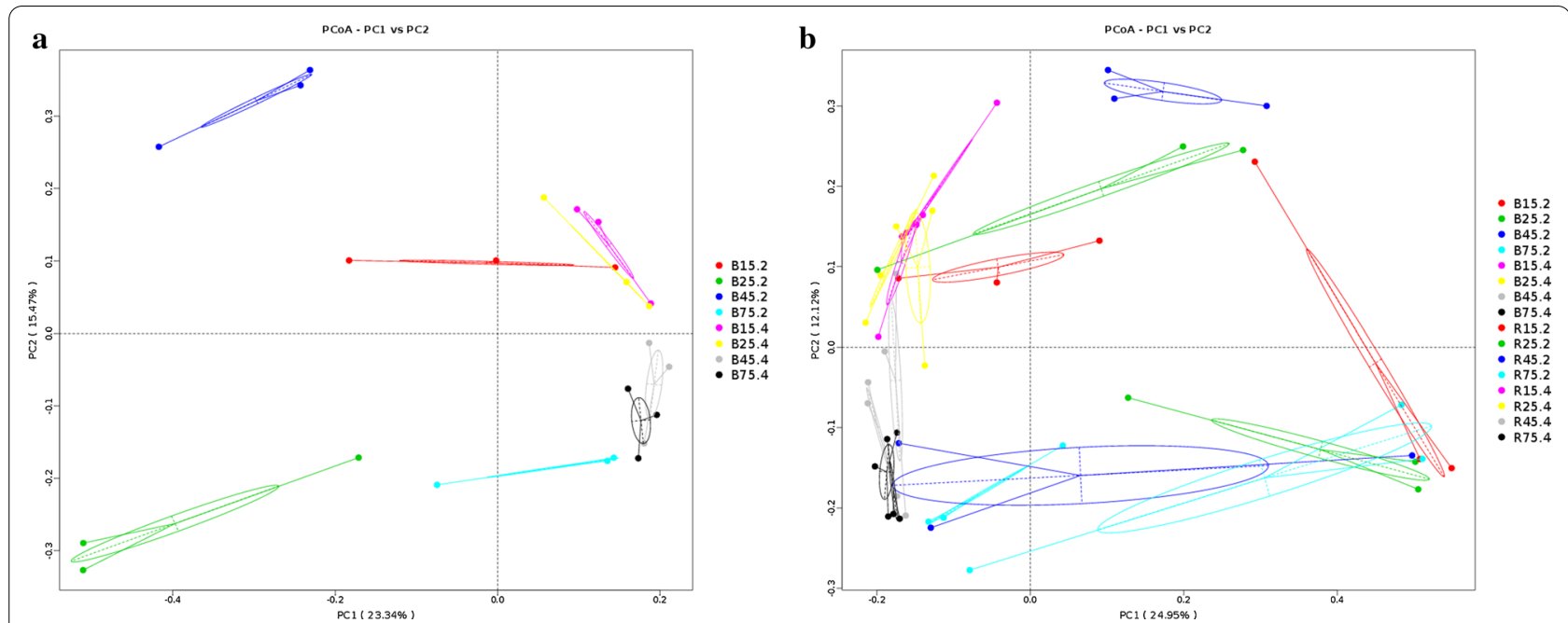

Fig. 3 Differences in principal component of gut microbial structures. Each colored point in the figure represents one sample. The distance between the two points indicates the difference of gut microbiota

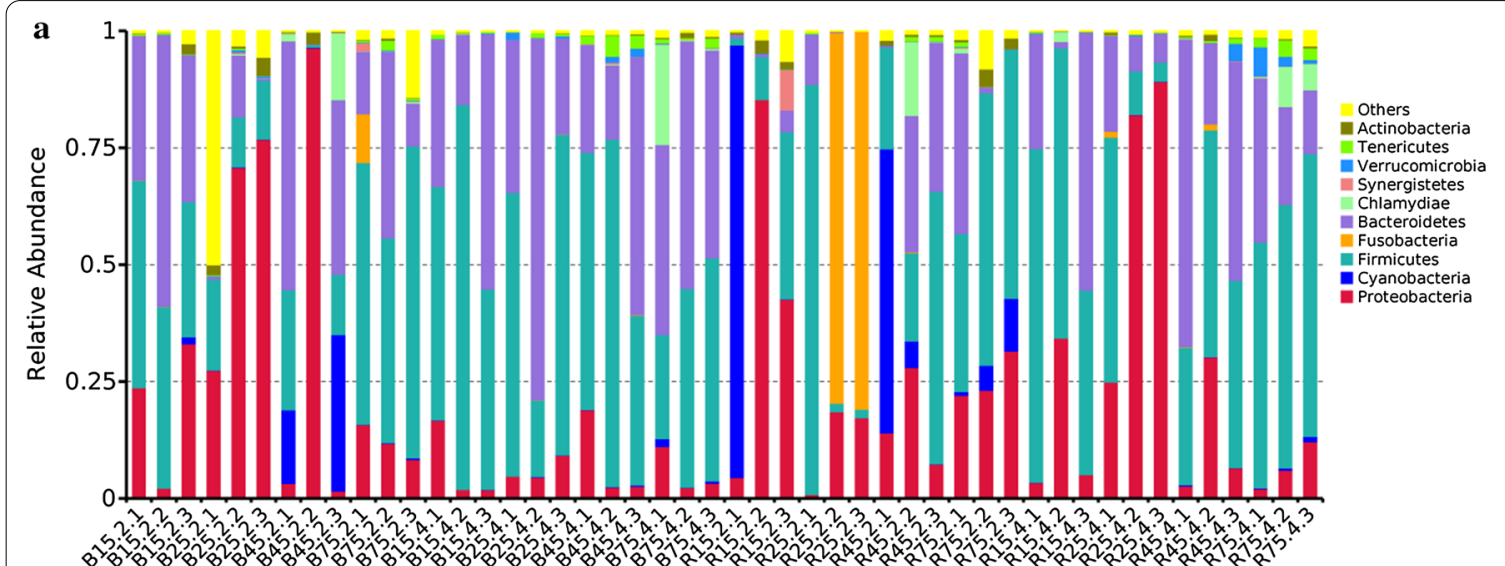

Sample Name

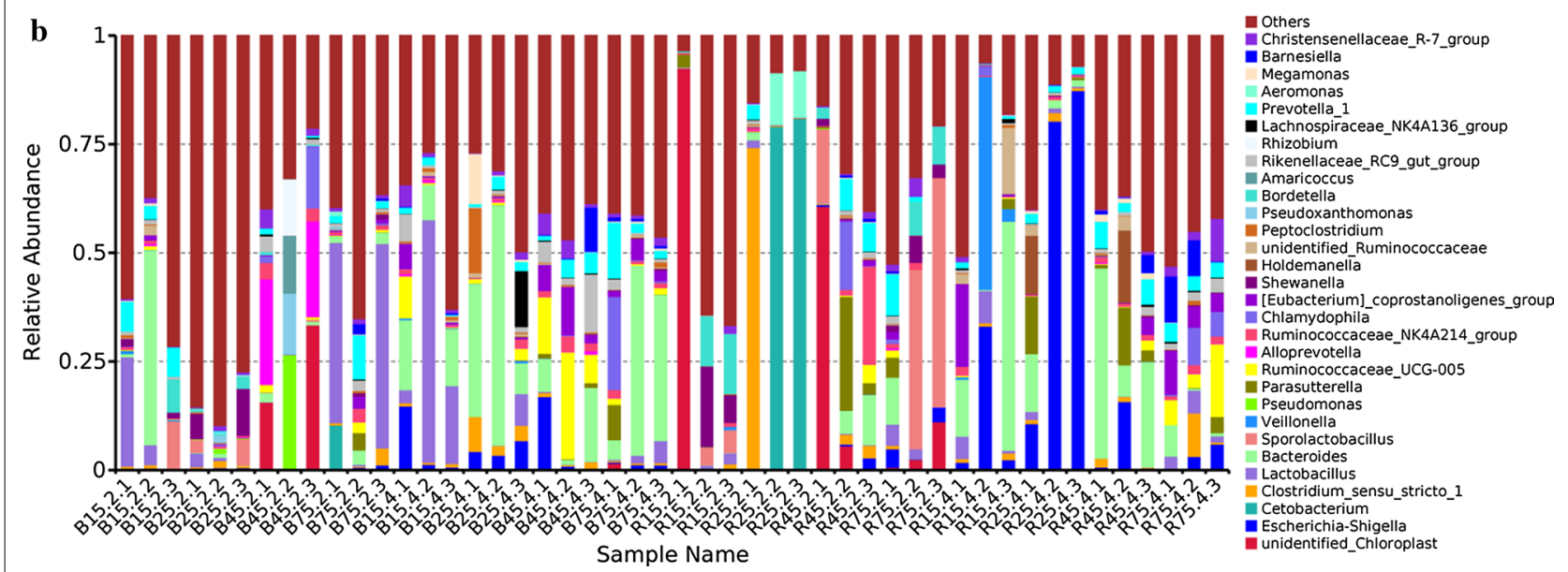

Fig. 4 Taxonomic distribution of different samples at the levels of phylum (a top ten) and genus (b top thirty). Each color-block represents the relative abundance of a bacterial taxon in a sample 


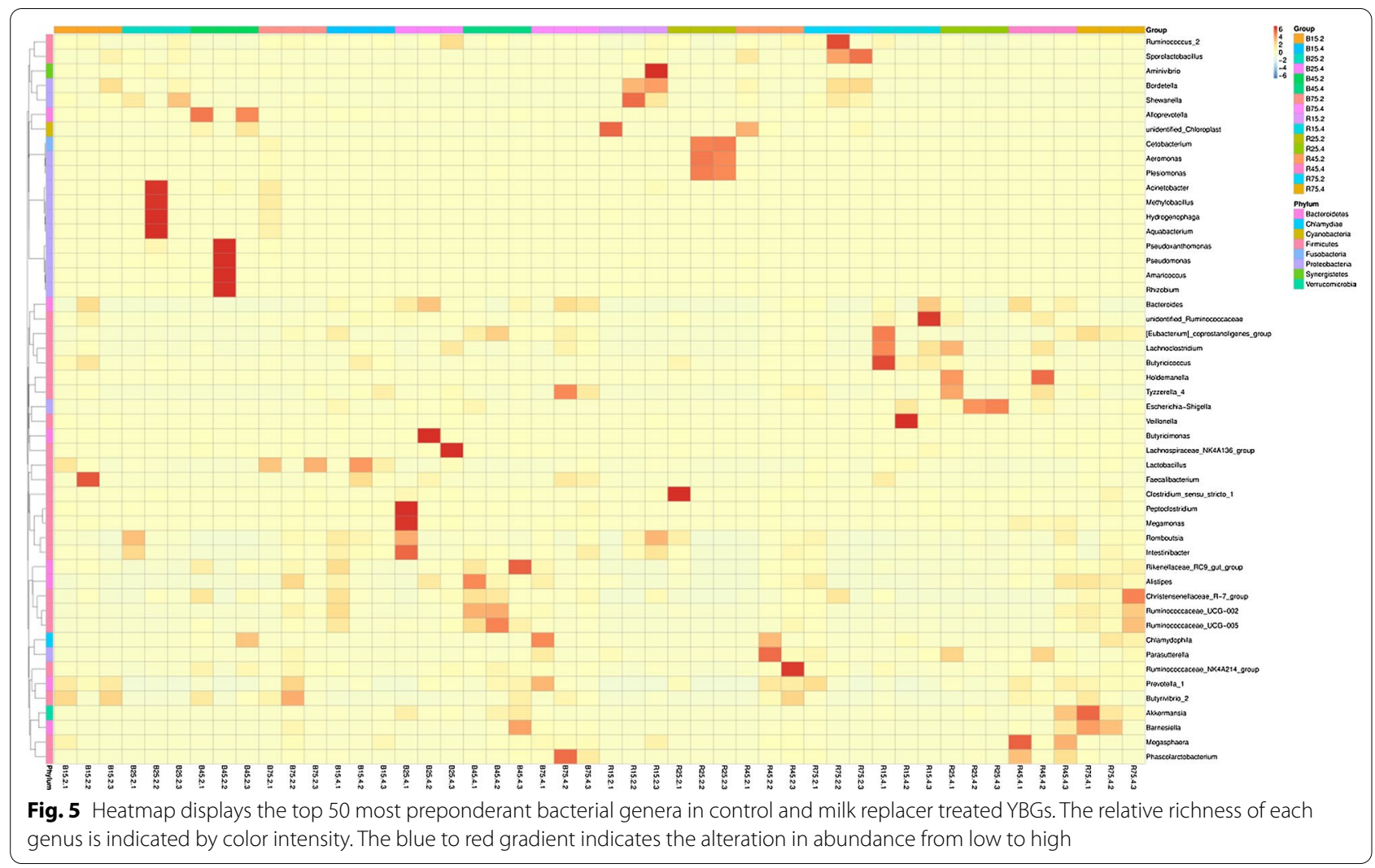

Eubacterium_nodatum_group in the B75.2 were higher than that in the B15.2. However, the relative abundances of Prevotellaceae_UCG_003 and Prevotella_1 in the B25.2, and Ruminococcus_2, Clostridium_ sensu_stricto_1 and Butyricicoccus in the B45.2 was lower than that in the B15.2, respectively (Fig. 6). The relative abundances of Clostridium_sensu_stricto_1 in the B25.4, Pseudobutyrivibrio, Lachnospiraceae NK3A20_group, Lachnospiraceae_UCG_001, Lachnospiraceae_AC2044_group and Bifidobacterium in the B45.4, Lachnospiraceae_XPB1014_group, Prevotellaceae_UCG_003, Prevotellaceae_UCG_001, Ruminococcus_1, Lachnospiraceae_AC2044_group, Lachnospiraceae_ND3007_group and Lachnospiraceae_UCG_001 in the B75.4 were significantly higher than that in the B15.4, respectively, whereas the relative abundances of Ruminiclostridium_9 in B25.4, and Ruminiclostridium in B45.4 were lower. Moreover, some pathogenic bacteria such as Moraxella, Streptococcus and Turicibacter in the B15.4 were significantly higher than that in the B45.4. Interestingly, the relative abundances of Lachnospiraceae, Ruminococcaceae and Ruminiclostridium in jejunum and Ruminococcaceae,
Eubacterium_ruminantium_group and Barnesiella in cecum significantly decreased and subsequently increased.

\section{Significant alterations in the gut microbial compositions of YBGs with the effect of milk replacer}

The phylum Proteobacteria was the most preponderant bacteria in the jejunum of 15-day-old YBGs treated by milk replacer, followed by the phyla Cyanobacteria and Firmicutes (Fig. 4). Moreover, the phylum Fusobacteria was much more abundant in the jejunum of 25-day-old YBGs treated by milk replacer than other phyla, whereas the phyla Firmicutes and Proteobacteria were the second and third most abundant, respectively. In contrast, the predominant bacteria in the jejunum of 45-day-old YBGs treated by milk replacer was the phylum Firmicutes, followed by the phyla Cyanobacteria and Bacteroidetes, which accounted for over $75 \%$ of all bacterial taxa. Additionally, the dominant phyla found in jejunum of milk replacer administration 75-day-old YGBs were also Proteobacteria, Firmicutes and Bacteroidetes, which were consistent with results in the control group. In the cecum of milk 

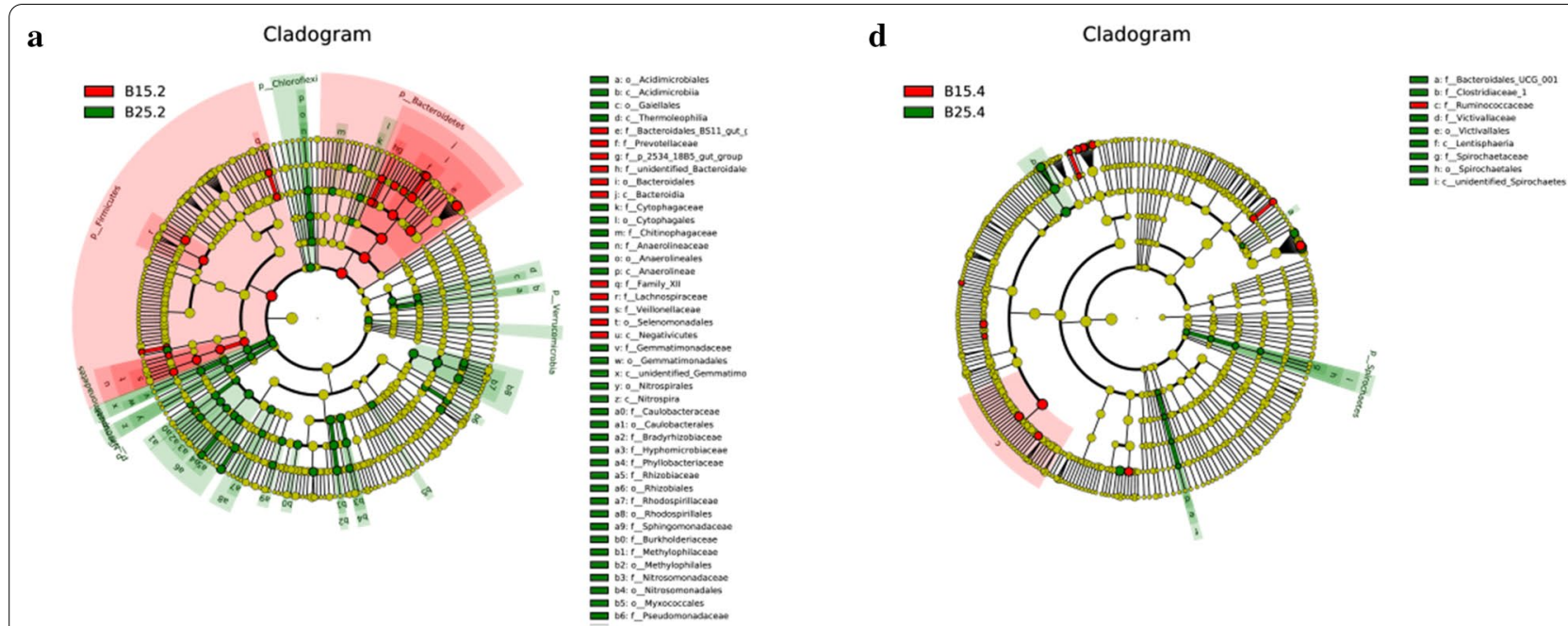

b

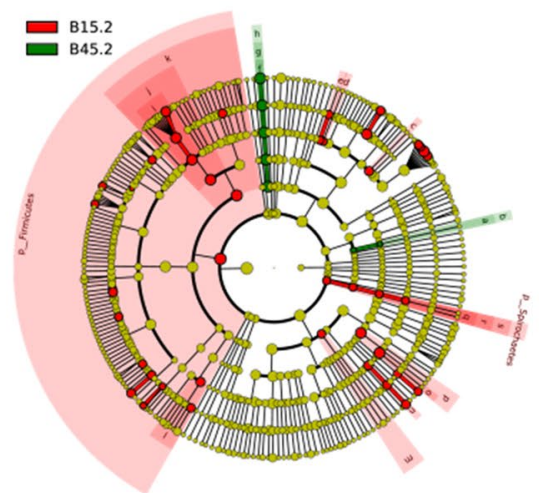

c

Cladogram

815.2
875.2

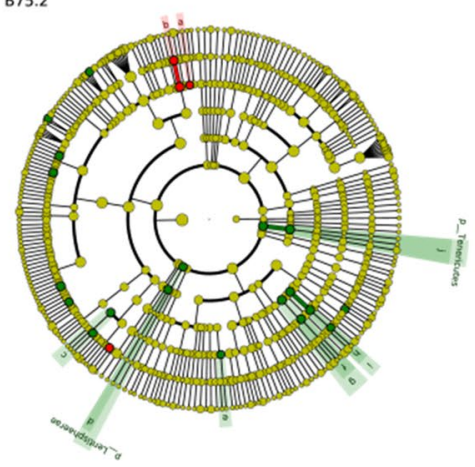

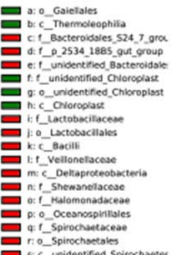

e

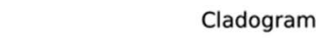

B 815.4
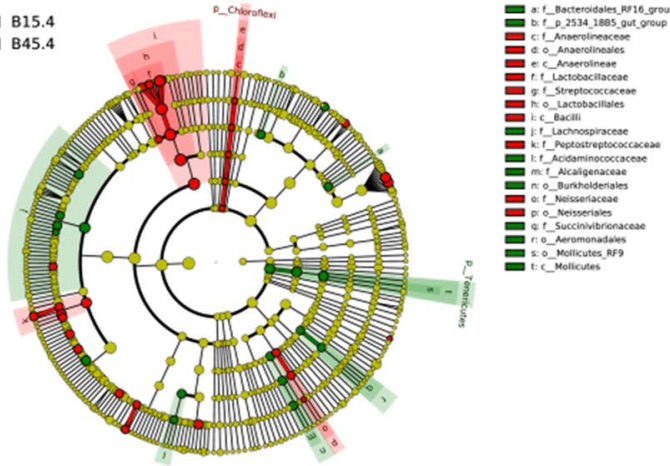

f

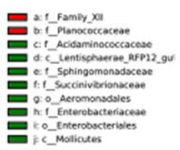

Cladogram

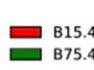

B75.4

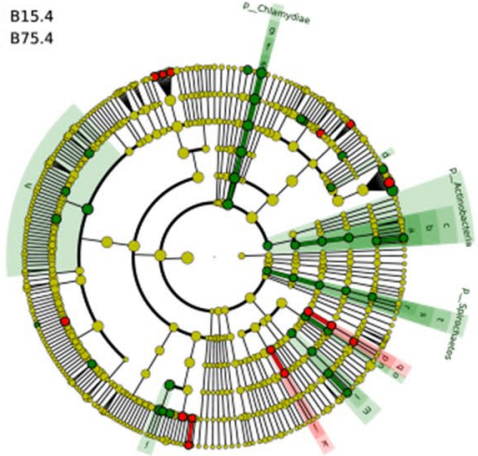

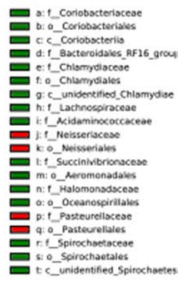

Fig. 6 Cladogram obtained from LEfSe analysis shows the different taxa in microbiota of YBGs with different ages. The colored circles from the inside to the outside represent the taxonomic levels of phylum, class, order, family, and genus. The yellow circles in the cladogram indicate the taxa with no significant differences

replacer administration YGBs, Proteobacteria, Firmicutes and Bacteroidetes were the three most preponderant phyla, which accounted for more than $93 \%$ of the total $16 \mathrm{~S}$ rRNA gene sequences. At the genus level, unidentified_Chloroplast (30.83\%) was the most dominant bacterium in the jejunum of 15-day-old YGBs treated 
by milk replacer, followed by Bordetella (8.51\%) and Shewanella (8.31\%), which together made up $47.65 \%$ of the bacterial composition. Cetobacterium (53.26\%), Clostridium_sensu_stricto_1 (24.75\%) and Aeromonas (7.59\%) were the most predominant bacteria in the jejunum of 25-day-old YGBs treated by milk replacer, while unidentified_Chloroplast (22.12\%), Parasutterella (9.73\%) and Ruminococcaceae_NK4A214_group (7.94\%) were observed to be predominant in the jejunum of 45-day-old YGBs treated by milk replacer. Sporolactobacillus (31.33\%) was the prevalent bacteria in the jejunum of 75-day-old YGBs treated by milk replacer, followed by Bordetella (5.49\%) and unidentified_Chloroplast (4.77\%). Bacteroides (22.00\%), Veillonella (17.50\%) and Escherichia-Shigella (12.49\%) were abundantly present in the cecum of 15-day-old YGBs treated by milk replacer, whereas the occurrence of Escherichia-Shigella (59.47\%), Bacteroides (5.51\%) and Parasutterella (4.63\%) were high in the cecum of 25-day-old YGBs treated by milk replacer. Bacteroides (25.09\%), Holdemanella (5.66\%) and Parasutterella (5.57\%) were the most prevalent bacteria in the cecum of 45-day-old YGBs treated by milk replacer, while Ruminococcaceae_ UCG-005 (8.56\%), Eubacterium_coprostanoligenes_ group (6.40\%) and Barnesiella (6.36\%) were the most abundant bacteria in the cecum of 75-day-old YGBs treated by milk replacer.

LEfSe analysis was performed to check the significant difference between control and milk replacer administration YBGs segments on the basis of taxa (phylum to genus) identification (Fig. 7). At the phylum level, the abundance of the Cyanobacteria, Actinobacteria and Proteobacteria were significantly decreased in R25.2 in comparison with B25.2. Additionally, Firmicutes was dramatically abundant in R75.4 than in the B75.4, whereas the abundance of Bacteroidetes was lower. At the genus level, Micrococcus level tended to be higher in the R15.2 than B15.2, whereas the Prevotellaceae_NK3B31_group, Prevotellaceae_UCG_001, Ruminococcus_1, Ruminiclostridium, Ruminococcaceae_UCG_005, Prevotellaceae_UCG_003, Porphyromonas and Prevotella_1 displayed the opposite trend. The relative abundance of Pseudobutyrivibrio, Ruminococcaceae_UCG_014, Brevundimonas, Flavobacterium, Turicibacter, Stenotrophomonas, Propionibacterium, Halomonas and Acinetobacter in the R25.2 were significantly lower than the B25.2. Meanwhile, the R45.2 was significantly enriched for Lachnospiraceae_NK3A20_group, Ruminococcus_2, Ruminococcaceae_UCG_014, Bifidobacterium and Lachnospiraceae_XPB1014_group in comparison with B45.2. Moreover, a comparison of the R75.2 and B75.2 displayed a significant increase in the abundance of Shewanella as well as a obvious decrease in the abundance of Acinetobacter and Enterococcus. The abundance of Clostridium_sensu_stricto_1 in the R15.4 was found relatively higher, whereas the levels of Tyzzerella_3, Eubacterium_ruminantium_group, Ruminococcaceae_UCG_014, Ruminococcaceae_UCG_013 and Ruminiclostridium_9 were lower as compared to B15.4.

The comparison of these identified taxa indicated that the B25.4 had a significantly higher abundance of Ruminiclostridium, Lachnospiraceae FE2018_group, Lachnospiraceae_UCG_010, Ruminococcaceae_UCG_005, Clostridium_sensu_stricto_1 and Butyricimonas than those of R25.4, while the relative abundance of Prevotellaceae_NK3B31 group was lower. Lachnospiraceae_UCG_010, Ruminiclostridium_9, Ruminococcaceae_UCG_009, Ruminiclostridium_5, Prevotellaceae_UCG_004, Ruminococcaceae_NK4A214_group, Christensenellaceae_R_7 group and Ruminococcaceae_UCG_005 levels tended to be higher in B45.4 than R45.4, while the Roseburia and unidentified_Lachnospiraceae showed the opposite trend. Moreover, R75.4 were characterized by high levels of Ruminococcaceae_UCG_005, Christensenellaceae_R_7_ group, Akkermansia, Ruminococcaceae_UCG_002, Ruminococcaceae_UCG_010, Ruminococcaceae UCG_013, Ruminiclostridium_5 and Ruminococcaceae UCG_009 compared with B75.4.

\section{Discussion}

Ruminant gut microbiota is an interactive and complex system, which play key roles in metabolism, immunity, nutrient absorption and intestinal mucosal barrier maintenance [16]. Recent studies revealed that gut microbiota was a vital barrier for host against the invasion and colonization of pathogenic bacteria, implying its crucial roles in the prevention and amelioration of diseases [17, 18]. However, the ruminant gut microbiota was dynamically varied and influenced by multiple factors such as feed, animal species and external environment $[19,20]$. Currently, the significance of milk replacer has been widely acknowledged as a result of its role in growth performance, immunity and health maintenance, but few reports have been published on the effect of milk replacer on gut microbiota of YBGs [21]. The present study investigated the effect of milk replacer administration on gut microbiota of YGBs and characterized the gut microbial shifts during the early period after birth.

Given feces cannot fully reflect the composition and diversity of gut microbial community, we selected the intestinal contents as the research object [22]. Age is an important factor affecting the structure and composition of gut microbial community [23]. Several studies have revealed that mammalian gut microbiota was normally 


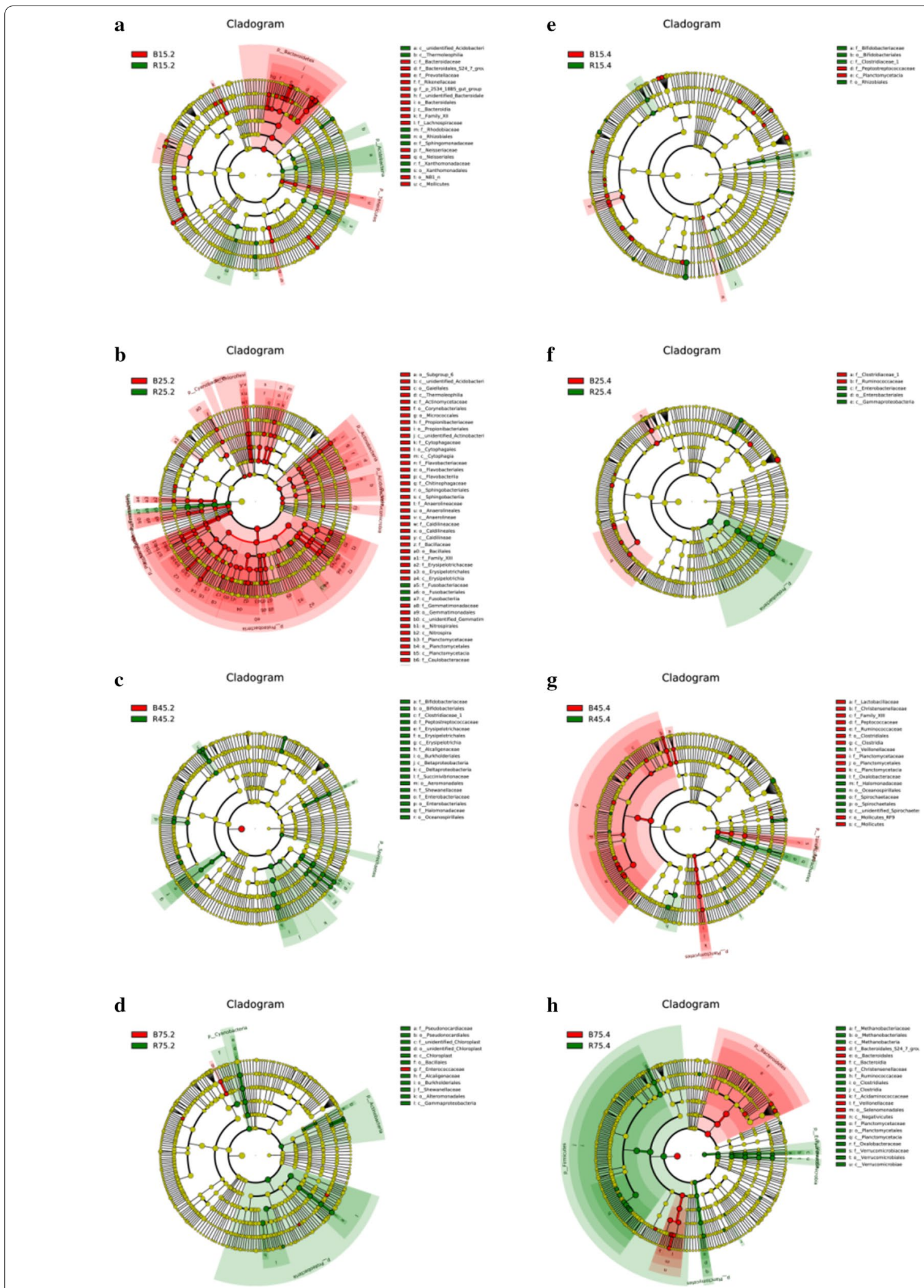

Fig. 7 LEfSe analysis indicated the differences in the relative abundance of control and milk replacer-treated groups. Cladogram reveals the phylogenetic distribution of gut bacterial community associated with control and milk replacer-treated groups. The taxa with no distinct differences was represented by the yellow circles 
affected by species, genotype and diet during development and reached stability at maturity [24, 25]. Wang et al. found that the differences of gut microbial diversity between juvenile and adult Boer goats were not significant [26]. Similarly, we observed that the alpha diversity of gut bacterial community did not change significantly with the age of YBGs. However, recent studies have revealed a dramatically increased alpha diversity of gut microbiota in musk deer, cattle and piglet with age, which was inconsistent with our observation in YBGs [27, 28]. We speculated that there may be differences in the composition and development of gut microbiota between different species and the gut microbiota of YBGs may reach a stable state at an earlier age. Remarkably, although microbial diversity was not dramatically different between different age groups, the proportion of some bacterial genera altered. We observed that the number and types of beneficial and functional bacteria increase with age, which was beneficial to realize the functional diversity of the gut. Moreover, some beneficial bacteria such as Lachnospiraceae, Ruminococcaceae, Ruminiclostridium, Eubacterium and Barnesiella significantly decreased and subsequently increase with age. This may be the result of the evolution of host towards a better structure during development.

Increasing evidence revealed the close relationship between gut microbial alterations and diet $[29,30]$. Ruminant gut microbiota in infancy is more sensitive to dietary changes due to the immature gastrointestinal tract. Hu et al. found that the alpha diversity of piglets was apparently decreased during the early period after weaning due to the sudden diet transition from breast milk to solid feed [28]. Our previous research has demonstrated that milk replacer administration could not change the diversity of rumen microbiota of earlyweaned YBGs, but the composition of the rumen microbiota has changed [14]. In the current study, we noticed that the milk replacer administration had no effects on the structure and diversity of the gut bacterial community of YBGs, which may be the results of the similarity in composition and physical structure of milk replacer and breast milk without causing stress response. Interestingly, although milk replacer administration and changing age did not alter the microbial diversity of YBGs, the percentage of some intestinal bacteria changed. At the phylum level, the ratio of Firmicutes in the gut microbiota of milk replacer-treated YBGs increased, whereas the ratio of Cyanobacteria, Actinobacteria and Proteobacteria decreased as compared to control YBGs. Remarkably, our previous study also indicated that milk replacer supplementation significantly reduced the abundance of Actinobacteria in the rumen of YBGs [14]. The Firmicutes is mainly responsible for the digestion of cellulose and its higher abundance in the intestinal environment contributes to meet the nutrition and energy requirements of animals during growth and development [31]. Moreover, Firmicutes contains large amount of gram-positive bacteria and some of them are regarded as beneficial bacteria, which contribute to against pathogenic invasion and maintain intestinal microflora balance [32]. Most members of Cyanobacteria can produce toxic cyanotoxin, which seriously threaten the health of human and animal [33]. It has been demonstrated that the relative abundance of Actinobacteria in the gut of diarrheal goat was dramatically increased [26]. In addition, the synergy of Actinobacteria with one partner or host can easily be transformed into a pathogenic interaction with another [34]. Proteobacteria mainly consists of many gram-negative bacteria such as Vibrio cholerae, Helicobacter pylori, Salmonella and Escherichia coli, which could result in diarrhea, gastritis, vomiting, gastrointestinal ulcers and even death, posing a great threat to animal health [35, 36].

Previous research has demonstrated that milk replacer supplementation ameliorated the gut microbiota of early-weaned yak and increased the abundance of bacteria involved in the utilization of fibrous and non-fibrous carbohydrates [37]. Consistent with previous study, this study also indicated that milk replacer administration improved the gut microbial composition of early-weaned YBGs, characterized by an increased abundance of some potentially beneficial gut bacteria and carbohydrate-degrading bacteria. Specifically, the proportion of Lachnospiraceae, Bifidobacterium, Prevotellaceae, Roseburia and Akkermansia in gut microbiota of milk replacer-treated YBGs increased, whereas the ratio of Porphyromonas, Brevundimonas, Flavobacterium, Stenotrophomonas, Propionibacterium, Acinetobacter, Enterococcus and Clostridium decreased. Lachnospiraceae was considered as potential probiotic in the rumen and intestine, displaying a negative correlation with intestinal inflammation [38]. Bifidobacterium, an important intestinal beneficial bacterium, displays multiple important physiological functions, such as anti-tumor, anti-aging and improving gastrointestinal function and immunity [39]. Importantly, Bifidobacterium can also improve the intestinal environment and inhibit the proliferation of pathogenic bacteria through producing antimicrobial peptides and organic acids [40]. Akkermansia was conducive to maintain gastrointestinal health and metabolic balance and decrease the risk of diabetes, obesity and inflammation [41]. Furthermore, Akkermansia was also involved in the improvement of gut barrier function and the regulation of immune homeostasis in the gut mucosa [42]. Prevotellaceae 
and Prevotella of the gut can degrade polysaccharide and high carbohydrate [43]. Both Porphyromonas and Brevundimonas were pathogenic bacteria, which can accelerate atherosclerosis and cause bacteremia, respectively [44, 45]. Flavobacterium, an opportunistic pathogen, can cause pneumonia, meningitis and sepsis as the host immunity decreases [46]. Stenotrophomonas, an emerging pathogens, was closely related to bacteremia, while Propionibacterium can cause Endocarditis, meningitis and dermatopathy [47, 48]. Acinetobacter, mainly inhabiting in the gastrointestinal tract, respiratory tract, skin and genitourinary tract, was opportunistic pathogen, which can result in pneumonia, endocarditis, bacteremia, as well as urinary and skin infections $[49,50]$. Enterococcus has been demonstrated to cause life-threatening sepsis, cardio-periostitis and meningitis [51]. Additionally, many antibiotics commonly used in the clinic failed in the treatment of Enterococcus infection, due to the inherent and acquired resistance [52]. This study conveyed a crucial message that milk replacer administration gradually ameliorated the gut microbial composition via increasing the proportion of beneficial and pathogenic bacteria. Clostridium was previously reported to play an important role in causing necrotizing enterocolitis [53]. Clostridium has also been shown to be closely associated with intestinal toxemia and diarrhea in ruminants and its toxins affects the host health via multiple pathways [54]. Furthermore, we also observed that some potential beneficial bacteria in jejunum (Ruminococcus and Ruminococcaceae) and cecum (Ruminococcaceae, Christensenellaceae and Ruminiclostridium) of the milk replacer-treated YBGs significantly decreased and subsequently increased. Ruminococcaceae displayed the ability to degrade cellulose and starch and was closely related to feed efficiency in lamb and cattle [55]. Moreover, Ruminococcaceae has long been thought to be potential beneficial bacterium because of the positive regulation of the immune system and intestinal environment [56]. Remarkably, recent studies demonstrated that the abundance of Ruminococcaceae in the gut microbiota was negatively correlated with liver cirrhosis, nonalcoholic fatty liver and increased intestinal permeability [57]. Ruminiclostridium, as a potential beneficial bacterium in the gut, not only involved in the positive regulation of the growth performance but also could secrete short-chain fatty acids, which was conducive to maintain functionality and morphology of intestinal epithelial cells and the regulation of intestinal microbiota balance [58]. Remarkably, some of these bacteria, such as Ruminococcus, Ruminococcaceae, Akkermansia, Eubacterium, Pseudobutyrivibrio, Roseburia and
Butyricimonas can also produce short-chain fatty acids (SCFAs), which could regulate intestinal permeability and maintain normal physiological functioning of the gut [59]. Moreover, SCFAs can also regulate energy intake through the brain-gut axis to alleviate the development of obesity and diabetes [60,61].

\section{Conclusion}

Taken together, this study indicated the gut microbial alterations in YBGs with the effect of age and milk replacer. Our results indicated that the difference in microbial diversity in YBGs with different ages was not significant, but the types and proportion of some beneficial bacteria on days 25,45 and 75 were higher than that on day 15 , which was conducive to the stability of the intestinal environment and realize the intestinal functional diversity. Moreover, milk replacer administration could improve the gut microbial composition and structure by increasing proportion of beneficial and harmful bacteria. These findings enriched the knowledge of gut microbiota in YBGs. Importantly, this study also expanded our understanding of the potential benefits of milk replacer and convey an important message that milk replacer may serve as a good applicant for ameliorating gut microbiota in early-weaned YBGs. However, several limitations presenting in this study need to be noticed, such as small sample size, dietary habit and individual variation.

\section{Acknowledgements \\ This work was supported by the Shandong Province Education Department "Animal Behavior Welfare and Healthy Breeding Technology Skills Innovation Team" Project, Shandong Province Agriculture Major Applied Technology Innovation Project and Shandong Province modern agricultural industry tech- nology system sheep innovation team Linyi experimental station.}

\section{Authors' contributions}

AYL and YY conceived and designed the experiments; SKQ, SJL, THJ, KL, ZQH and YZL contributed sample collection and reagents preparation. All authors read and approved the final manuscript.

Availability of data and materials Yes.

\section{Declarations}

Ethics approval and consent to participate

Animal experiments were performed under the approval of Ethics Committee of the Linyi University

\section{Consent for publication}

Yes.

\section{Competing interests}

The authors declare that they have no competing interests.

\section{Author details}

${ }^{1}$ College of Agriculture and Forestry Science, Linyi University, Linyi, China. ${ }^{2}$ College of Veterinary Medicine, Huazhong Agricultural University, Wuhan 430070, China. ${ }^{3}$ Institute of Traditional Chinese Veterinary Medicine, College of Veterinary Medicine, Nanjing Agricultural University, Nanjing 210095, China. ${ }^{4}$ Linyi Academy of Agricultural Sciences, Linyi 276012, China. 
Received: 27 December 2020 Accepted: 20 March 2021

Published online: 31 March 2021

\section{References}

1. Lynch SV, Pedersen O. The human intestinal microbiome in health and disease. N Engl J Med. 2016;375(24):2369-79.

2. Desselberger U. The mammalian intestinal microbiome: composition, interaction with the immune system, significance for vaccine efficacy, and potential for disease therapy. Pathogens. 2018;7(3):57.

3. Smith B, Bode S, Petersen BL, Jensen TK, Pipper C, Kloppenborg J, Boye M, Krogfelt KA, Molbak L. Community analysis of bacteria colonizing intestinal tissue of neonates with necrotizing enterocolitis. BMC Microbiol. 2011;11:73.

4. Aziz Q, Dore J, Emmanuel A, Guarner F, Quigley EM. Gut microbiota and gastrointestinal health: current concepts and future directions. Neurogastroenterol Motil. 2013:25(1):4-15.

5. Dabke K, Hendrick G, Devkota S. The gut microbiome and metabolic syndrome. J Clin Invest. 2019;129(10):4050-7.

6. Wang Y, Li A, Zhang L, Waqas M, Mehmood K, lqbal M, Muyou C, Li Z, Lian Y, Sizhu S, et al. Probiotic potential of Lactobacillus on the intestinal microflora against Escherichia coli induced mice model through highthroughput sequencing. Microb Pathog. 2019;137:103760.

7. Kootte RS, Vrieze A, Holleman F, Dallinga-Thie GM, Zoetendal EG, de Vos WM, Groen AK, Hoekstra JB, Stroes ES, Nieuwdorp M. The therapeutic potential of manipulating gut microbiota in obesity and type 2 diabetes mellitus. Diabetes Obes Metab. 2012;14(2):112-20.

8. Guo W, Li Y, Wang L, Wang J, Xu Q, Yan T, Xue B. Evaluation of composition and individual variability of rumen microbiota in yaks by $16 \mathrm{~S}$ rRNA highthroughput sequencing technology. Anaerobe. 2015;34:74-9.

9. Chapman CE, Erickson PS, Quigley JD, Hill TM, Bateman HN, Suarez-Mena FX, Schlotterbeck RL. Effect of milk replacer program on calf performance and digestion of nutrients with age of the dairy calf. J Dairy Sci. 2016:99(4):2740-7.

10. Toral PG, Chilliard Y, Rouel J, Leskinen H, Shingfield KJ, Bernard L. Comparison of the nutritional regulation of milk fat secretion and composition in cows and goats. J Dairy Sci. 2015;98(10):7277-97.

11 Amdi C, Pedersen M, Klaaborg J, Myhill LJ, Engelsmann MN, Williams AR, Thymann T. Pre-weaning adaptation responses in piglets fed milk replacer with gradually increasing amounts of wheat. Br J Nutr. 2020. https://doi.org/10.1017/S0007114520004225.

12. Santos A, Giraldez FJ, Valdes C, Trevisi E, Lucini L, Frutos J, Andres S. Milk replacer restriction during early life impairs the live body weight and progesterone patterns of ewe lambs during the replacement period. J Dairy Sci. 2018;101(9):8021-31.

13. Bhatt RS, Tripathi MK, Verma DL, Karim SA. Effect of different feeding regimes on pre-weaning growth rumen fermentation and its influence on post-weaning performance of lambs. J Anim Physiol Anim Nutr. 2009;93(5):568-76.

14. Han Z, Li A, Pei L, Li K, Jin T, Li F, Wang Z, Lv S, Li Y. Milk replacer supplementation ameliorates growth performance and rumen microbiota of early-weaning Yimeng black goats. Front Vet Sci. 2020;7:572064.

15. Zhang Y, Wang K, Liu J, Zhu H, Qu L, Chen H, Lan X, Pan C, Song X. An 11-bp indel polymorphism within the CSN1S1 gene is associated with milk performance and body measurement traits in Chinese goats. Animals. 2019;9(12):1114

16. Wu HJ, Wu E. The role of gut microbiota in immune homeostasis and autoimmunity. Gut Microbes. 2012;3(1):4-14

17. Turnbaugh PJ, Ley RE, Mahowald MA, Magrini V, Mardis ER, Gordon JI. An obesity-associated gut microbiome with increased capacity for energy harvest. Nature. 2006:444(7122):1027-31.

18. Carding SR, Davis N, Hoyles L. Review article: the human intestinal virome in health and disease. Aliment Pharmacol Ther. 2017:46(9):800-15.

19. Li K, Mehmood K, Zhang H, Jiang X, Shahzad M, Dong X, Li J. Characterization of fungus microbial diversity in healthy and diarrheal yaks in Gannan region of Tibet Autonomous Prefecture. Acta Trop. 2018;182:14-26.

20. Zhang L, Jiang X, Li A, Waqas M, Gao X, Li K, Xie G, Zhang J, Mehmood K, Zhao $S$, et al. Characterization of the microbial community structure in intestinal segments of yak (Bos grunniens). Anaerobe. 2020;61:102115.
21. Stanley CC, Williams CC, Jenny BF, Fernandez JM, Bateman HN, Nipper WA, Lovejoy JC, Gantt DT, Goodier GE. Effects of feeding milk replacer once versus twice daily on glucose metabolism in Holstein and Jersey calves. J Dairy Sci. 2002;85(9):2335-43.

22. Zeng Y, Zeng D, Zhang Y, Ni X, Tang Y, Zhu H, Wang H, Yin Z, Pan K, Jing B. Characterization of the cellulolytic bacteria communities along the gastrointestinal tract of Chinese Mongolian sheep by using PCR-DGGE and real-time PCR analysis. World J Microbiol Biotechnol. 2015;31(7):1103-13.

23. Hu J, Nie Y, Chen J, Zhang Y, Wang Z, Fan Q, Yan X. Gradual changes of gut microbiota in weaned miniature piglets. Front Microbiol. 2016;7:1727.

24. Poroyko V, Morowitz M, Bell T, Ulanov A, Wang M, Donovan S, Bao N, Gu S, Hong L, Alverdy JC, et al. Diet creates metabolic niches in the "immature gut" that shape microbial communities. Nutr Hosp. 2011;26(6):1283-95.

25. Zhao W, Wang Y, Liu S, Huang J, Zhai Z, He C, Ding J, Wang J, Wang H, Fan $W$, et al. The dynamic distribution of porcine microbiota across different ages and gastrointestinal tract segments. PLoS ONE. 2015;10(2):e117441.

26. Wang Y, Zhang H, Zhu L, Xu Y, Liu N, Sun X, Hu L, Huang H, Wei K, Zhu R. Dynamic distribution of gut microbiota in goats at different ages and health states. Front Microbiol. 2018;9:2509.

27. Hu X, Liu G, Shafer A, Wei Y, Zhou J, Lin S, Wu H, Zhou M, Hu D, Liu S. Comparative analysis of the gut microbial communities in forest and alpine musk deer using high-throughput sequencing. Front Microbiol. 2017;8:572.

28. Jami E, Israel A, Kotser A, Mizrahi I. Exploring the bovine rumen bacterial community from birth to adulthood. ISME J. 2013;7(6):1069-79.

29. Mayengbam S, Chleilat F, Reimer RA. Dietary vitamin B6 deficiency impairs gut microbiota and host and microbial metabolites in rats. Biomedicines. 2020;8(11):469.

30. Sun B, Vatanen T, Jayasinghe TN, McKenzie E, Murphy R, O'Sullivan JM. Desacetyl-alpha-MSH and alpha-MSH have sex specific interactions with diet to influence mouse gut morphology, metabolites and microbiota. Sci Rep. 2020;10(1):18957.

31. Sun B, Wang X, Bernstein S, Huffman MA, Xia DP, Gu Z, Chen R, Sheeran LK, Wagner RS, Li J. Marked variation between winter and spring gut microbiota in free-ranging Tibetan Macaques (Macaca thibetana). Sci Rep. 2016;6:26035

32. Garneau JE, Tremblay DM, Moineau S. Characterization of 1706, a virulent phage from Lactococcus lactis with similarities to prophages from other Firmicutes. Virology. 2008;373(2):298-309.

33. Carmichael WW. Cyanobacteria secondary metabolites - the cyanotoxins. J Appl Bacteriol. 1992;72(6):445-59.

34. Miao V, Davies J. Actinobacteria: the good, the bad, and the ugly. Antonie Van Leeuwenhoek. 2010;98(2):143-50.

35. Yang H, Xiao Y, Gui G, Li J, Wang J, Li D. Microbial community and short-chain fatty acid profile in gastrointestinal tract of goose. Poult Sci. 2018:97(4):1420-8.

36. Huang Z, Kraus VB. Does lipopolysaccharide-mediated inflammation have a role in OA? Nat Rev Rheumatol. 2016;12(2):123-9.

37. Wu S, Cui Z, Chen X, Wang P, Yao J. Changed caecalmicrobiota and fermentation contribute to the beneficial effects of early weaning with Alfalfa Hay, starter feed, and milk replacer on the growth and organ development of yak calves. Animals. 2019;9(11):921.

38. Zhao L, Zhang Q, Ma W, Tian F, Shen H, Zhou M. A combination of quercetin and resveratrol reduces obesity in high-fat diet-fed rats by modulation of gut microbiota. Food Funct. 2017;8(12):4644-56.

39. Sivan A, Corrales L, Hubert N, Williams JB, Aquino-Michaels K, Earley ZM, Benyamin FW, Lei YM, Jabri B, Alegre ML, et al. Commensal Bifidobacterium promotes antitumor immunity and facilitates anti-PD-L1 efficacy. Science. 2015;350(6264):1084-9.

40 Underwood MA, Arriola J, Gerber CW, Kaveti A, Kalanetra KM, Kananurak A, Bevins CL, Mills DA, Dvorak B. Bifidobacterium longum subsp. infantis in experimental necrotizing enterocolitis: alterations in inflammation, innate immune response, and the microbiota. Pediatr Res. 2014;76(4):326-33.

41. Liu C, Li XH, Chen YX, Cheng ZH, Duan QH, Meng QH, Tao XP, Shang B, Dong HM. Age-related response of rumen microbiota to mineral salt and effects of their interactions on enteric methane emissions in cattle. Microb Ecol. 2017;73(3):590-601.

42. Derrien M, Belzer C, de Vos WM. Akkermansiamuciniphila and its role in regulating host functions. Microb Pathog. 2017;106:171-81.

43. De Filippo C, Cavalieri D, Di Paola M, Ramazzotti M, Poullet JB, Massart S, Collini S, Pieraccini G, Lionetti P. Impact of diet in shaping gut microbiota 
revealed by a comparative study in children from Europe and rural Africa. Proc Natl Acad Sci USA. 2010;107(33):14691-6.

44. Li L, Messas E, Batista EJ, Levine RA, Amar S. Porphyromonas gingivalis infection accelerates the progression of atherosclerosis in a heterozygous apolipoprotein E-deficient murine model. Circulation. 2002;105(7):861-7.

45. Chi CY, Fung CP, Wong WW, Liu CY. Brevundimonas bacteremia: two case reports and literature review. Scand J Infect Dis. 2004;36(1):59-61.

46. Nematollahi A, Decostere A, Pasmans F, Haesebrouck F. Flavobacterium psychrophilum infections in salmonid fish. J Fish Dis. 2003;26(10):563-74.

47. Muder RR, Harris AP, Muller S, Edmond M, Chow JW, Papadakis K, Wagener MW, Bodey GP, Steckelberg JM. Bacteremia due to Stenotrophomonas (Xanthomonas) maltophilia: a prospective, multicenter study of 91 episodes. Clin Infect Dis. 1996;22(3):508-12.

48. Clayton JJ, Baig W, Reynolds GW, Sandoe J. Endocarditis caused by Propionibacterium species: a report of three cases and a review of clinical features and diagnostic difficulties. J Med Microbiol. 2006;55(Pt 8):981-7.

49. Livermore DM, Woodford N. The beta-lactamase threat in Enterobacteriaceae, Pseudomonas and Acinetobacter. Trends Microbiol. 2006;14(9):413-20.

50. Lima WG, Brito J, Da CNW. Ventilator-associated pneumonia (VAP) caused by carbapenem-resistant Acinetobacter baumannii in patients with COVID-19: two problems, one solution? Med Hypotheses. 2020;144:110139.

51. Mohanty S, Dhawan B, Kapil A, Das BK, Pandey P, Gupta A. Brain abscess due to Enterococcus avium. Am J Med Sci. 2005;329(3):161-2.

52. Arias CA, Murray BE. The rise of the Enterococcus: beyond vancomycin resistance. Nat Rev Microbiol. 2012;10(4):266-78.

53. Zhou Y, Shan G, Sodergren E, Weinstock G, Walker WA, Gregory KE. Longitudinal analysis of the premature infant intestinal microbiome prior to necrotizing enterocolitis: a case-control study. PLoS ONE. 2015;10(3):e118632

54. Pepin J, Valiquette L, Alary ME, Villemure P, Pelletier A, Forget K, Pepin $\mathrm{K}$, Chouinard D. Clostridium difficile-associated diarrhea in a region of
Quebec from 1991 to 2003: a changing pattern of disease severity. CMAJ. 2004;171(5):466-72.

55. Zhao J, Yao Y, Li D, Xu H, Wu J, Wen A, Xie M, Ni Q, Zhang M, Peng G, et al. Characterization of the gut microbiota in six geographical populations of Chinese Rhesus Macaques (Macaca mulatta), implying an adaptation to high-altitude environment. Microb Ecol. 2018;76(2):565-77.

56. Kong F, Hua Y, Zeng B, Ning R, Li Y, Zhao J. Gut microbiota signatures of longevity. Curr Biol. 2016;26(18):R832-3.

57. Shang Q, Shan X, Cai C, Hao J, Li G, Yu G. Dietary fucoidan modulates the gut microbiota in mice by increasing the abundance of Lactobacillus and Ruminococcaceae. Food Funct. 2016;7(7):3224-32.

58. Tan J, McKenzie C, Potamitis M, Thorburn AN, Mackay CR, Macia L. The role of short-chain fatty acids in health and disease. Adv Immunol. 2014;121:91-119.

59. Smith PM, Howitt MR, Panikov N, Michaud M, Gallini CA, BohloolyY M, Glickman JN, Garrett WS. The microbial metabolites, shortchain fatty acids, regulate colonic Treg cell homeostasis. Science. 2013;341(6145):569-73.

60. LeBlanc JG, Chain F, Martin R, Bermudez-Humaran LG, Courau S, Langella P. Beneficial effects on host energy metabolism of short-chain fatty acids and vitamins produced by commensal and probiotic bacteria. Microb Cell Fact. 2017;16(1):79.

61. Tappenden KA, Thomson AB, Wild GE, McBurney MI. Short-chain fatty acids increase proglucagon and ornithine decarboxylase messenger RNAs after intestinal resection in rats. JPEN J Parenter Enteral Nutr. 1996;20(5):357-62.

\section{Publisher's Note}

Springer Nature remains neutral with regard to jurisdictional claims in published maps and institutional affiliations.
Ready to submit your research? Choose BMC and benefit from:

- fast, convenient online submission

- thorough peer review by experienced researchers in your field

- rapid publication on acceptance

- support for research data, including large and complex data types

- gold Open Access which fosters wider collaboration and increased citations

- maximum visibility for your research: over 100M website views per year

At BMC, research is always in progress.

Learn more biomedcentral.com/submissions 